\title{
Méthodes de mesure du métabolisme respiratoire chez les animaux aquatiques
}

\author{
par
}

ERic Pattee

Il existe trois façons d'étudier l'influence du milieu environnant sur un organisme entier:

On peut tenir compte du changement que produit la variation du milieu extérieur sur le comportement, l'activité, ou parfois l'aspect de l'organisme.

On peut aussi, après avoir mesuré, dans des conditions déterminées, le niveau léthal de chaque facteur (température léthale, tension d'asphyxie), modifier ces conditions et suivre la variation du niveau léthal qui en résulte.

On peut enfin évaluer le métabolisme respiratoire de l'organisme. Ce métabolisme est généralement considéré comme le critère le plus sensible et le plus fidèle de l'activité intérieure de l'organisme. C'est en quelque sorte le meilleur indicateur de l'intensité avec laquelle vit celui-ci.

Le métabolisme respiratoire se traduit par deux phénomènes: une absorbtion d'oxygène et un rejet de gaz carbonique. ${ }^{1}$ Chez les animaux à respiration aquatique, que nous envisagerons ici, les gaz utilisé et rejeté le sont sous forme dissoute dans l'eau.

La façon la plus simple de mesurer ce métabolisme consiste à disposer le sujet dans une enceinte close où on le laisse respirer et d'évaluer la quantité d'O $\mathrm{O}_{2}$ qui disparaît ou la quantité de $\mathrm{CO}_{2}$ qui apparaît. C'est le procédé dit ,e n mili e u c on fin é’. Le mode de fermeture le plus employé pour les récipients respiratoires est le bouchon de verre rodé, conique ou plat. Il a l'avantage de per-

1) Une certaine quantité de chaleur est produite, correspondant approximativement aux oxydations pour lesquelles est utilisé l' $\mathrm{O}_{2}$ absorbé (tant qu'il n'y a pas anaérobiose). Cette thermogénèse, telle qu'elle a été mesurée en 1954 par PRAT permet donc de se faire une idée du métabolisme de l'animal étudié. 
mettre un jaugeage précis du volume intérieur du récipient qui demeure toujours le même. Si la partie rodée n'est pas assez large, si le rodage n'est pas assez précis et qu'il ne soit pas graissé, des fuites et une diffusion de l'oxygène peuvent se produire. Trop d'auteurs ont employé une couche d'huile de paraffine pour isoler de l'air le milieu où respirent les animaux. LALlEmand a pourtant signalé dès 1932 que l'oxygène est plus soluble dans cette huile que dans l'eau elle-même et qu'il pénètre dans le milieu soi-disant isolé dès le début des expériences. L'huile ne fait que retarder la diffusion de 1 ' $\mathrm{O}_{2}$. Tenant compte de ces phénomènes, Philipson (1954) a dû construire des courbes de diffusion de $\mathrm{l}^{\prime} \mathrm{O}_{2}$ afin de corriger les résultats de ses mesures.

On peut également organiser une circulation d'eau traversant l'enceinte où respire l'animal et comparer l'eau à l'entrée et à la sortie du système. C'est le procédé e n milie u renou velé, dont l'avantage réside en ce qu'il maintient la constance du milieu, empêchant l'asphyxie progressive et l'accumulation de substances nuisibles. Il est donc d'autant plus indiqué que la durée de la mesure est plus longue. Mais sa précision est moins bonne que celle du procédé en milieu confiné car de grands volumes d'eau entrent en jeu et les quantités relatives d'O $\mathrm{O}_{2}$ disparu et de $\mathrm{CO}_{2}$ apparu sont d'autant plus faibles que la masse liquide est plus importante.

Il est possible de réunir en trois groupes les méthodes de mesure de ces échanges gazeux:

1) L es $\mathrm{M}$ é $\mathrm{t}$ hodes $\mathrm{Ch}$ i miques. Elles permettent de doser la teneur en $\mathrm{O}_{2}$ ou en $\mathrm{CO}_{2}$ du milieu avant et après le séjour de l'animal.

La méthode de LuND (1919) consiste à capter le $\mathrm{CO}_{2}$ par de la baryte et à doser ensuite la quantité de $\mathrm{CO}_{3} \mathrm{Ba}$ obtenue. SAyLE a utilisé en 1928 une adaptation de cette méthode à des animaux aquatiques. De l'air barbotte dans la cellule respiratoire, se chargeant donc du $\mathrm{CO}_{2}$ produit, puis il passe dans la baryte. Le milieu peut donc presque être qualifié de renouvelé, sa teneur en $\mathrm{CO}_{2}$ étant pratiquement nulle et la teneur en $\mathrm{O}_{2}$ presque constante. Ces méthodes de dosage du $\mathrm{CO}_{2}$ présentent cependant un inconvénient: la présence de carbonates et de bicarbonates dans les eaux naturelles fausserait les mesures de $\mathrm{CO}_{2}$, suivant la réaction

$$
\mathrm{CO}_{3} \mathrm{Na}_{2}+\mathrm{CO}_{2}+\mathrm{H}_{2} \mathrm{O} \leftrightarrows 2 \mathrm{CO}_{3} \mathrm{HNa}
$$

Aussi Sayle utilise-t-elle de l'eau physiologique composée de $\mathrm{ClNa}, \mathrm{ClK}, \mathrm{Cl}_{2} \mathrm{Ca}$. Ce n'est pas là le milieu naturel des animaux.

La méthode chimique la plus employée est celle de WINKLER (1888), qui a été contrôlée par Theriault (1925), Pomeroy \& 
KirschmaN (1945). Elle permet de doser l'O$_{2}$ dissous en remplaçant dans la solution un atome d'oxygène par une molécule d'iode. L'iode est ensuite titré à l'hyposulfite. C'est une très bonne méthode, sûre et assez précise tant que l'eau ne contient pas de nitrites, mais longue et fastidieuse.

Le problème à résoudre est celui de l'introduction des divers réactifs tout en maintenant la solution à l'abri de l'air jusqu'à la formation de l'iode. Plusieurs techniques ont été mises au point, utilisant les récipients suivants:

Un erlen rodé que l'on bouche après adjonction de chaque réactif (WINKLER 1924, DusSART \& FRANCIS-BOEUF 1949).

Le tube de Nicioux, contenant 25 cc d'eau environ, réduction et amélioration de l'erlen précédent par sa forme en $\mathrm{Y}$ et la bille de verre qu'il renferme. C'est seulement une fois le tube bouché que les réactifs sont mis en présence les uns des autres. La bille permet d'agiter la solution pour y parvenir.

Les pipettes d'Albert LeVY $(10 \mathrm{à} 150 \mathrm{cc})$ où l'eau à étudier se trouve dans un tube, constamment isolée de l'air par un robinet à chaque extrémité. Cette technique est la plus précise.

Les seringues de VAN DAM (1935) ou KROGH (1935) pour les petits volumes jusqu'à $1,5 \mathrm{cc}$ dont Fox et ses collaborateurs ont été les principaux utilisateurs (Fox \& WINGFIELD 1938). Ces seringues présentent l'inconvénient suivant: de l'eau de capillarité, en quantité souvent variable, se trouve entre le corps de la seringue et le piston. Le jaugeage de certaines seringues nous a montré que le volume interne variait de 4 à $5 \%$, ce qui aurait provoqué dans le résultat des mesures d'oxygène une erreur inacceptable. Il faut donc que l'espace contenant l'eau de capillarité soit aussi régulier et aussi réduit que possible. Nous ne sommes arrivé à de bons résultats (précision de 0,1 à $0,5 \%$ pour une eau normalement oxygénée) qu'en employant des seringues de haute précision (marque Impec par exemple) ou parfois en graissant à la graisse de silicones celles qui donnaient des résultats trop variables.

Le capillaire qui termine la seringue doit être fin pour réduire l'espace mort et empêcher la diffusion d' $\mathrm{O}_{2}$, mais point trop car il rendrait impossible l'aspiration des acides phosphorique ou sulfurique, de consistance visqueuse. Un diamètre interne de $1 \mathrm{~mm}$ semble représenter le minimum possible.

Il est enfin nécessaire que le précipité d'hydroxyde manganique soit aussi fin que possible. Un précipité grossier obstrue l'entrée de la seringue à la façon d'un tampon d'ouate et rend toute agitation de la solution impossible. Or il est bien connu (Fox \& WINGFIELD 1938) que le précipité est d'autant plus fin que les réactifs sont plus dilués. On ne peut pas songer à diluer la solution d'iodure qui doit 
au contraire être aussi concentrée que possible (POMEROY \& KirSCHMAN 1945), mais on peut faire varier à volonté la concentration de la solution de $\mathrm{Cl}_{2} \mathrm{Mn}$. Nous obtenons de bons résultats avec une solution de $\mathrm{Cl}_{2} \mathrm{Mn}$ à $3 \%$, au lieu de $10 \%$ et plus comme on la prépare pour la méthode de WINKLER habituelle.

Les méthodes chimiques sont le plus souvent utilisées pour des mesures en milieu confiné. On dose la quantité d' $\mathrm{O}_{2}$ avant et après l'expérience. Néanmoins, certains auteurs ont opéré en milieu renouvelé (CLAUSEN 1936 sur des poissons, WaLSHE 1947 sur Chironomus, BLÄsING 1953 sur des Planaires). Ils prélevaient à intervalles réguliers des échantillons de l'eau à la sortie des cellules respiratoires et dosaient l' $\mathrm{O}_{2}$. Connaissant la teneur en $\mathrm{O}_{2}$ à l'entrée et le débit, ils déduisaient la consommation des animaux.

2) Les Méthodes Electriques. Les principales consistent à mesurer par polarographie la teneur en $\mathrm{O}_{2}$ de l'eau avant et après le séjour de l'animal. On peut employer soit un polarographe muni d'une électrode à gouttes de mercure (par exemple la technique de MoORe, Morris \& OKun 1948 au celle de RotTHAUWe 1958), soit un polarographe muni d'une électrode solide (électrode de platine comme celle de GIGUERE \& LAUZIER 1945, électrode d'or comme celle de LoNGMUIR 1954 et 1957 ou de AMBÜHL 1959), soit plus simplement un microampèremètre et une électrode à gouttes de mercure de lumière plus large $(0,8 \mathrm{~mm})$ (BRIGGS, DYKE \& KNOwles 1958).

Ces méthodes sont comparables aux méthodes chimiques, mais leur emploi est plus rapide, une fois l'appareil étalonné. Elles sont moins sûres, par suite des déviations qui peuvent se produire (SPOOR 1948, Wood 1953) et demandent des vérifications fréquentes qui peuvent se faire par comparaison avec les résultats fournis par la méthode chimique de WINKLER. L'appareillage est complexe et onéreux. Il ne faut pas perdre de vue que le mercure est un poison et qu'il n'est pas question de faire respirer les animaux dans la cellule même où l'on fait le dosage au moyen d'une électrode à gouttes de mercure. Un transvasement à l'abri de l'air est toujours nécessaire.

Une intéressante adaptation de ces méthodes est celle utilisée par MaNN en 1958 sur la sangsue Erpobdella. Un courant d'eau lent et régulier (milieu renouvelé) traverse la chambre respiratoire puis la cellule du polarographe, permettant l'enregistrement continu de la teneur en $\mathrm{O}_{2}$. Du point de vue des conditions où se trouve l'animal, c'est une méthode idéale. Mais MaNN a éprouvé de grandes difficultés techniques: le mucus des sangsues engluait les électrodes, il était impossible d'ajouter de produits destinés à supprimer les maxima des courbes. L'auteur pense cependant avoir obtenu de bons résultats.

Il est actuellement possible de réaliser l'inscription automatique 
des résultats si l'on opère sur un volume d'eau et donc sur un nombre d'animaux suffisants (BRIGgS, Davies, DYKE \& KNOWLes 1957 et BRIGGS, DYKe \& KNOwles 1959). Grâce à un système d'horlogerie, le polarographe de ces auteurs enregistre la tension d'O$_{2}$ pendant 3 minutes toutes les demi-heures et ne demande qu'une séance d'entretien hebdomadaire.

Nous citerons pour mémoire le „Diaferomètre" de PUNT (1950 et 1956), basé sur la variation de la conduction thermique dans un mélange gazeux suivant sa teneur en $\mathrm{CO}_{2}$. Il n'a pas été, à notre connaissance, utilisé pour l'étude d'animaux aquatiques.

3) Les $M$ éthodes de $M$ esure des $Q u a n t i t e ́ s$ Gazeus es:

Scholander, van Dam, ClafF \& Kanwisher (1955) ont mis au point une méthode gazométrique susceptible de rendre des services dans certaines conditions, en particulier lorsque l'eau est polluée. Le principe en est l'extraction des gaz en solution et la mesure de leur volume à une température et à une pression connues, avant et après absorbtion de $\mathrm{l}^{\prime} \mathrm{O}_{2}$ par le pyrogallol.

Il ne semble pas que les autres méthodes dites gazométriques soient utilisables actuellement pour l'étude des animaux aquatiques.

Les méthodes manométriques, dans le sens large tel que l'emploie Dixon dans le titre de son précieux petit traité, en 1951, sont par contre très souvent utilisées.

Le principe est le suivant: capter le $\mathrm{CO}_{2}$ par de la soude et mesurer la disparition de $\mathrm{l}^{\prime} \mathrm{O}_{2}$ consommé par l'animal. Il est ensuite possible de neutraliser la soude par de l'acide sulfurique et donc de libérer le $\mathrm{CO}_{2}$ que l'on mesure alors. Ces méthodes peuvent être divisées en trois groupes, suivant les conditions dans lesquelles on évalue la disparition de $\mathrm{l}^{\prime} \mathrm{O}_{2}$ :

a) Mesure de la différence de pression à volume constant; appareil de WarbuRg. C'est le plus simple et le plus employé. Une des branches du manomètre est ouverte à l'air, si bien que le niveau est influencé par les variations de température et de pression atmosphérique. Les corrections à faire intervenir sont indiquées par la déviation d'appareils vides identiques aux précédents et disposés dans les mêmes conditions.

b) Mesure de la différence de pression et de volume simultanément; appareil de BarcrofT. Cet appareil est formé d'un manomètre dont chaque branche est reliée à une cellule: d'un côté la cellule où respire l'animal, de l'autre la cellule de compensation. L'ensemble est clos, donc indépendant des variations atmosphériques et contient un certain volume d'air de chaque côté du manomètre, il est donc indépendant des petites variations de température.

Les appareils de WARBURG et de BARCROFT nécessitent un jau- 
geage précis du volume interne, d'où l'on tire les constantes (Dixon 1951). Le calcul est assez complexe dans le cas de l'appareil de BARCROFT où la pression et le volume changent à la fois.

c) Mesure de la différence de volume à pression constante; méthode dite volumétrique; appareils de WINTERSTEIN, HALDANE et plus récemment SCHOLANDER \& EDWARDS (1942). La verrerie employée est plus complexe, comprenant d'un côté du manomètre la cellule respiratoire et une burette spéciale, de l'autre la cellule de compensation. La burette permet d'injecter dans le système une quantité d'air mesurée et telle qu'elle ramène le manomètre à son niveau de départ, égale donc à la quantité d'O $\mathrm{O}_{2}$ absorbée par l'animal. On lit ainsi directement la quantité d' $\mathrm{O}_{2}$ disparue, les calculs sont simplifiés (ils consistent à ramener cette quantité d' $\mathrm{O}_{2}$ aux conditions N.T.P.). Il n'y a pas de correction à faire intervenir, l'appareil étant compensé. Le volume de la cellule n'intervient pas dans le calcul du résultat, ce qui permet de le modifier à son gré. Il est possible d'injecter dans l'appareil à l'aide de la burette, non plus de l'air mais de l' $\mathrm{O}_{2}$. Le milieu où se trouve l'animal aura alors une teneur constante en $\mathrm{O}_{2}$ et en $\mathrm{CO}_{2}$. On peut donc le qualifier de renouvelé quant à ces deux facteurs importants.

Ces techniques manométriques se prêtent à la construction de microrespiromètres capillaires aussi simples qu'ingénieux, tels celui de LANE, TIERNEY \& HeNNACY (1954). Un tube capillaire gradué où l'on dispose un index de liquide coloré sert de manomètre et se termine d'un côté par un renflement de 6 à $250 \mathrm{~mm}^{3}$ qui est la cellule respiratoire, de l'autre par un rodage auquel peut s'adapter une cellule de compensation. On dépose dans la cellule respiratoire une goutte de soude d'un côté, une goutte d'eau contenant l'animal de l'autre, et on ferme au moyen d'une lamelle graissée. La lecture des déplacements de l'index permettra de calculer la consommation d' $\mathrm{O}_{2}$ de l'animal.

Il existe de nombreuses autres microméthodes (technique du Ludion, micromètre à levier optique par exemple), décrites notamment par ToBIAs (1943).

Un dernier perfectionnement à ces méthodes manométriques a été apporté par BROwN en 1954. Son plongeur est le seul appareil manométrique permettant l'inscription automatique des résultats. La cellule est en communication avec un sac de plastique déformable

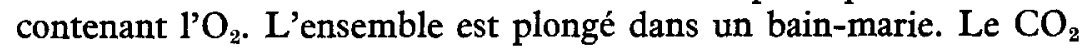
est absorbé à mesure qu'il est rejeté par l'animal. La consommation $\mathrm{d}^{\prime} \mathrm{O}_{2}$ produit une diminution du volume du sac, donc une augmentation de la densité du système, et le plongeur descend. Le mouvement est enregistré sur un cylindre. L'appareil, dans sa première forme, n'était pas compensé: il demandait une correction pour les 
variations de température et de pression. Mais l'inertie des leviers inscripteurs des plongeurs-témoins pouvait être une source importante d'erreur. Plus tard, en 1957, Brown fut amené à modifier l'appareil et à inclure les plongeurs par groupe de 4 dans une enceinte à pression constante.

Lors de la construction d'un de ces appareils et en particulier d'un de ceux du type volumétrique, il importe de tenir compte des trois points suivants.

$1^{\circ}$ point. Les gaz respirés sont dissous. Or les manomètres mesurent des gaz dans leur phase gazeuse. Il faut donc un équilibre parfait entre l' $\mathrm{O}_{2}$ et le $\mathrm{CO}_{2}$ de l'eau et de l'air. Certains auteurs considèrent que l'animal agite suffisamment le milieu liquide pour que l'équilibre se réalise à sa surface (CALHOon \& ANGERER 1953). BRown ne prévoit aucun moyen d'accélérer les échanges entre l'air et l'eau dans son respiromètre.

Les appareils de BARCROFT et de WARBURG sont destinés à être secoués dans leur bain-marie à raison d'environ une oscillation par seconde, afin de brasser l'eau qu'ils contiennent et de multiplier ses contacts avec le gaz du manomètre. Il est difficile de considérer que le métabolisme d'un animal ainsi balotté est voisin du métabolisme en milieu naturel. Le respiromètre de SCHOLANDER offre une meilleure solution: cet auteur introduit une hélice dans la cellule respiratoire. L'hélice, traversant un joint étanche d'agar, est mue par un petit moteur situé au-dessus du respiromètre. Elle agite efficacement la surface de l'eau. Avec cet appareil, nous avons maintes fois constaté la diminution de la consommation d' $\mathrm{O}_{2}$ dès que nous arrêtions la rotation de l'hélice et une recrudescence de cette soi-disant consommation lorsque nous remettions l'hélice en marche. C'est une preuve que celle-ci est nécessaire à la diffusion des gaz. De même, Dixon (1951) insiste sur le fait suivant: si les résultats fournis par les manomètres de BARCROFT et de WARBURG dépendent de la fréquence des oscillations, c'est l'indice que la diffusion des gaz devient un facteur limitant et que les mesures sont faussées. Dans le choix d'une méthode, il faut donc tenir compte de l'agitation produite par l'animal et déterminer si elle suffit ou non à la diffusion de l'O$_{2}$.

De même, la surface de la soude doit être assez grande pour capter rapidement tout le $\mathrm{CO}_{2}$ de la phase gazeuse, sinon il convient de l'augmenter en utilisant des rouleaux de papier-filtre imbibés de soude (Dixon 1951).

$2^{\circ}$ point. Le manomètre dévie lentement dans un sens ou dans l'autre tant que l'équilibre thermique entre les différentes parties de l'appareil n'est pas absolu. Cet équilibre peut se réaliser en 30 minutes (pour le microrespiromètre de LANE) ou en 6 heures environ 
(pour le respiromètre de ScHOLANDER avec cellule de $30 \mathrm{cc}$ ). L'équilibre est d'autant plus rapide que les volumes sont plus petits.

A l'opposé, l'appareil est d'autant plus sensible que la cellule de compensation est plus grande. Il faut donc choisir une moyenne pour le volume de la cellule de compensation, en fonction de ce que l'on désire: grande sensibilité ou équilibre rapide.

$3^{\circ}$ point. Nous avons toujours constaté une certaine déviation des manomètres, même s'ils ne contiennent pas d'animaux en expérience. Nous pensons en trouver la cause dans le B.O.D. de l'eau. C'est ainsi que nous mesurons une déviation plus forte lorsque l'eau utilisée provient d'un ruisseau de plaine que lorsqu'elle provient d'une source du Jura. On est donc conduit de nouveau à choisir une moyenne entre ces deux extrêmes: un grand volume d'eau pour diminuer le confinement de l'animal et se rapprocher des conditions naturelles, ou un volume d'eau aussi faible que possible pour réduire le B.O.D. et la déviation de l'appareil. Ou encore, si l'on veut: obtenir la mesure approchée du métabolisme habituel de l'animal ou obtenir la mesure exacte d'un métabolisme anormal.

En résumé, pour que l'appareil soit sensible, il faut une petite cellule respiratoire et une grande cellule de compensation.

Pour que l'appareil soit fidèle, il faut un petit volume d'eau, efficacement agitée.

Pour que l'équilibre thermique se réalise rapidement, il faut que tous les volumes d'air, d'eau et de verre soient petits.

Enfin il ne faut jamais oublier que les conditions faites à l'animal (espace vital, agitation) sont les plus importantes et qu'il est inutile de mesurer avec précision un métabolisme anormal.

Les comparaisons entre ces différentes mé$\mathrm{t}$ h o d e s sont rares. Et lorsqu'une espèce a été étudiée deux fois, on s'aperçoit souvent qu'il n'y a rien à tirer de la confrontation des deux valeurs obtenues, car celles-ci concernent des températures différentes ou sont exprimées l'une en fonction du poids sec, l'autre en fonction du poids frais (c'est le cas de la comparaison citée par АмвüHL à la page 222 de son article paru en 1959). Il faudrait pourtant s'attendre à des chiffres quelque peu différents suivant que l'on utilise l'appareil de WARBURG où l'animal est secoué une fois par seconde, la méthode de WINKLER où l'animal est confiné dans un petit volume stagnant, ou bien celle de MaNN où l'eau s'écoule continuellement.

Nous n'avons trouvé qu'une seule fois des résultats comparables pour le même animal (Chironomus) chez deux auteurs différents: EWER (1942) qui employait la méthode chimique de WinkLER, a mesuré à $17^{\circ}$ un métabolisme de $188 \mathrm{~mm}^{3}$ par gramme et par heure. 
EDWARDS (1958) utilisait un appareil du type WARBURG qu'il secouait à raison de 86 périodes par minute. Il a obtenu, par interpolation à $17^{\circ}$, le chiffre de $280 \mathrm{~mm}^{3}$. Le résultat de la méthode chimique correspond donc aux $67 \%$ de celui de la méthode manométrique.

Nous avons comparé les résultats de la méthode chimique utilisant les seringues de vaN DAM, et ceux de la méthode volumétrique de Scholander. Dans la première, l'eau est stagnante; dans la seconde, elle est agitée par une hélice. Le milieu est entièrement confiné dans le premier cas; la teneur en $\mathrm{CO}_{2}$ est nulle et la teneur en $\mathrm{O}_{2}$ pratiquement constante dans le second cas. Les tableaux I et II donnent nos résultats concernant deux espèces qui vivaient ensemble dans le même ruisseau de plaine, au Nord de Lyon.

\section{Tableau I.}

Comparaison des méthodes chimique et manométrique pour la mesure de la consommation d' $\mathrm{O}_{2}$ chez Dendrocoelum lactaeum Müller, exprimée en $\mathrm{mm}^{3}$ par heure et par individu. Température du biotope d'origine et des expériences: $12^{\circ}, 5$.

\begin{tabular}{l|cccc|c}
\hline Lots d'animaux & I & II & III & IV & Moyennes \\
\hline \multirow{3}{*}{ Méthode chimique } & 5,05 & 4,72 & 3,99 & 2,97 & \\
& 4,78 & 3,96 & 3,74 & 3,35 & 4,04 \\
& 5,20 & 4,06 & 3,38 & 3,28 & \\
Méthode manométrique & 7,77 & 5,33 & 3,76 & 4,07 & \\
& 8,02 & 5,59 & 3,80 & 3,81 & 5,33 \\
& 7,78 & 5,12 & 4,46 & 3,79 & \\
& 8,00 & 5,05 & 4,46 & 4,39 &
\end{tabular}

TABleau II.

Comparaison des méthodes chimique et manométrique pour la mesure de la consommation d' $\mathrm{O}_{2}$ chez Planaria gonocephala Dugès, exprimée en $\mathrm{mm}^{3}$ par heure et par individu. Température du biotope d'origine et des expériences: $12^{\circ}, 5$.

\begin{tabular}{l|ccccc|c}
\hline Lots d'animaux & I & II & III & V & Moyennes \\
\hline Méthode chimique & 1,74 & 3,00 & 2,02 & 4,42 & 1,54 & 2,54 \\
\hline \multirow{2}{*}{ Méthode manométrique } & 1,61 & 3,16 & 1,84 & 3,92 & 1,45 & 2,36 \\
& 1,53 & 2,94 & 1,98 & 3,72 & 1,46 & 2,36
\end{tabular}

Chez Dendrocoelum, la moyenne trouvée par la méthode chimique correspond environ aux $75 \%$ de celle par la méthode manométrique. L'analyse de variance pour la comparaison de ces deux moyennes donne un rapport hautement significatif. Les limites de 
l'intervalle de confiance de la différence sont de $1,29 \pm 0,46$ soit 0,83 et $1,75 \mathrm{cc}$.

Chez Planaria gonocephala au contraire, la méthode chimique a donné des chiffres significativement plus élevés que ceux de la méthode manométrique (la proportion mesurée est d'environ $110 \%$ ).

Nous voyons donc que Dendrocoelum, plus active, bien plus excitable lorsqu'on la stimule, réagit très différemment suivant la méthode employée. Elle respire plus intensément lorsque l'eau est agitée (courant et vibrations). Planaria gonocephala au contraire, animal plus lent, qui a tendance à se rétracter lorsqu'on le stimule, semble être moins sensible à la méthode employée et respirer moins intensément lorsque l'eau est agitée.

Crenobia alpina est une Planaire de montagne que nous avons récoltée dans le Jura à près de $850 \mathrm{~m}$ d'altitude. Comme pour Dendrocoelum, la moyenne que nous avons mesurée par la méthode chimique représente les $75 \%$ de celle par la méthode manométrique (Tableau III). Malgré le faible nombre des mesures, la différence des moyennes est significative.

\section{TABLEAU III.}

Comparaison des méthodes chimique et manométrique pour la mesure de la consommation d' $\mathrm{O}_{2}$ chez Crenobia alpina Dana, exprimée en $\mathrm{mm}^{3}$ par heure et par individu. Température du biotope d'origine et des expériences: $5^{\circ}, 5$.

\begin{tabular}{|c|c|c|c|}
\hline Lots d'animaux & I & II & Moyennes \\
\hline Méthode chimique & $\begin{array}{l}0,213 \\
0,220\end{array}$ & $\begin{array}{l}0,094 \\
0,105\end{array}$ & 0,158 \\
\hline Méthode manométrique & $\begin{array}{l}0,236 \\
0,258 \\
0,355\end{array}$ & $\begin{array}{l}0,158 \\
0,172 \\
0,122\end{array}$ & 0,217 \\
\hline
\end{tabular}

Les trois Vers précédents sont des animaux d'eau courante. Le milieu stagnant de la méthode chimique est anormal pour eux. Nous avons donc étudié un Invertébré d'eau stagnante. Cloëon dipterum, qui vivait dans les bassins du Jardin Botanique du Parc de la Tête d'Or à Lyon. Pour réduire l'agitation de l'eau dans le respiromètre de SCHOLANDER, nous avions disposé un écran de plastique perforé dans la cellule entre les animaux et l'hélice. Les deux moyennes ne sont cependant pas identiques, mais diffèrent significativement d'après l'analyse de variance. La méthode chimique a donné une moyenne correspondant aux $120 \%$ de celle de la méthode manométrique (Tableau IV).

Les résultats fournis par une seule méthode ne sont donc pas 
TABLEAU IV.

Comparaison des méthodes chimique et manométrique pour la mesure de la consommation d' $\mathrm{O}_{2}$ chez Cloëon dipterum L., exprimée en $\mathrm{mm}^{3}$ par heure et par individu. Température du biotope d'origine et des expériences: $3^{\circ}$.

\begin{tabular}{l|cc|c}
\hline Lots d'animaux & I & II & Moyennes \\
\hline \multirow{2}{*}{ Méthode chimique } & 0,426 & 0,396 & 0,369 \\
& 0,352 & 0,303 & \\
\hline \multirow{3}{*}{ Méthode manométrique } & 0,348 & 0,304 & \\
& 0,309 & 0,274 & 0,307 \\
& 0,300 & 0,304 &
\end{tabular}

valables dans l'absolu, mais ne le sont que comparativement à d'autres résultats fournis par la même méthode. La plus grande prudence s'impose lors de l'utilisation de résultats obtenus par deux méthodes différentes. Pour toute mesure de métabolisme où l'on désire pénétrer le comportement de l'animal dans la nature, il faut tenir compte en premier lieu des conditions qui lui sont offertes pendant l'expérience.

Fox \& Simmonds (1933) s'en sont aperçus et pour éliminer cette influence du milieu et rendre leurs résultats tous comparables entre eux, ils ont anesthésié les Arthropodes étudiés, de façon à mesurer ce qu'on pense être le métabolisme de base. La respiration est alors diminuée de plus de moitié.

Nous avons déjà signalé (Wautier \& Pattee 1955) que, chez certaines larves d'Insectes, on obtient des résultats très différents suivant la nature du substrat:

Ecdyonurus, larve d'Ephémère pétricole, respire en moyenne: sur fond de verre $1410 \mathrm{~mm}^{3} \mathrm{~d}^{\prime} \mathrm{O}_{2}$ par heure et par gramme sec, sur fond de cailloux $856 \mathrm{~mm}^{3} \mathrm{~d}^{\prime} \mathrm{O}_{2}$ par heure et par gramme sec, soit les $60 \%$ du chiffre précédent. L'analyse de variance avec blocs casualisés montre que la différence de ces deux moyennes est hautement significative.

Ephemera, larve fouisseuse, respire en moyenne: sur fond de verre $1130 \mathrm{~mm}^{3} \mathrm{~d}^{\prime} \mathrm{O}_{2}$ par heure et par gramme sec, sur fond de cailloux $1265 \mathrm{~mm}^{3}$, sur fond de sable $368 \mathrm{~mm}^{3}$, soit environ les $30 \%$ du chiffre correspondant au fond de verre. L'analyse de variance indique une différence hautement significative entre la consommation sur fond de sable d'une part et la consommation sur des substrats anormaux pour Ephemera (verre lisse ou cailloux) d'autre part. Il n'y a aucune différence significative entre ces deux derniers substrats.

La diminution du métabolisme lorsque l'animal se trouve dans 
un milieu qui rappelle son habitat normal est comparable à celle provoquée par une anesthésie. Or toutes les méthodes ne permettent pas de disposer du sable au fond des récipients. Les méthodes en milieu renouvelé sont toujours les meilleures, mais leur emploi est délicat, l'appareillage encombrant et les conditions (température, oxygénation, turbulence) souvent difficiles à contrôler. Parmi les méthodes en milieu confiné, celle de WINKLER reste la plus souple, permettant d'utiliser tous les volumes de $2 \mathrm{cc}$ à 1 litre ou plus (sous réserve que soit connu le volume d'eau à la disposition de l'animal) et tous les substrats. Cette eau peut être stagnante ou agitée au moyen d'une hélice qui opère à l'abri de l'air, sous un joint étanche. $\mathrm{La}$ seule condition est de choisir un volume d'eau convenable pour que la respiration de l'animal abaisse la tension d' $\mathrm{O}_{2}$ d'une quantité qui soit mesurable $(1 / 4$ ou $1 / 2$ de la quantité totale d' $\mathrm{O}_{2}$ dissous) sans porter préjudice à l'occupant et sans influencer sa respiration par contre-coup.

Parmi les méthodes manométriques, seule convient la méthode volumétrique qui permet de disposer sable ou cailloux dans la cellule sans modifier les constantes de l'appareil. Dans le respiromètre de Scholander, l'eau est agitée par une hélice. Il est cependant possible d'étudier des animaux d'eau stagnante en les protégeant par des écrans de plastique perforés. Le but recherché est l'agitation de l'interface air-eau plus que l'agitation de l'eau dans sa masse.

L a pré c i s i on des mét hodes, à l'intérieur de certaines limites, ne constitue qu'une préoccupation de second ordre, bien moins importante que les conditions faites à l'animal. Les variations individuelles entre différents sujets dépassent toujours de beaucoup les variations dûes à l'erreur de la méthode. C'est ainsi que BERG, LUMBYE \& OCKELMANN (1958) ont pu calculer que les variations dans leurs résultats sur le métabolisme d'Ancylus correspondaient à un écart-type de $11 \%$, dont $6 \%$ pour la précision de la méthode et $9 \%$ pour les différences individuelles entre animaux. Nous-même avons calculé, d'après 20 mesures sur le métabolisme de plusieurs séries de 2 larves de Libellula à $12^{\circ}$, les chiffres suivants: la variation globale (coefficient de variation) s'élève à $36 \%$, la variation dûe à la méthode à $2,5 \%$ et la variation dûe aux animaux à $35,9 \%$, soit pratiquement $36 \%$.

Il est très difficile de citer un chiffre traduisant la précision d'une de ces méthodes, l'erreur relative étant éminemment variable suivant le résultat des mesures. En voici un exemple:

La méthode de WinkLer permet de mesurer, non pas la quantité $\mathrm{d}^{\prime} \mathrm{O}_{2}$ disparue, mais la différence entre la tension $\mathrm{d}^{\prime} \mathrm{O}_{2}$ avant et après le séjour de l'animal. La précision de chaque résultat dépend 
donc de cette différence. Chaque lecture de la burette à hyposulfite se fait avec une erreur absolue de $0,025 \mathrm{cc}$. L'ensemble des deux lectures se fait done avec une erreur de $0,05 \mathrm{cc}$. Or la différence entre les deux lectures varie généralement entre 5 et $0,5 \mathrm{cc}$, d'où une erreur relative comprise entre 1 et $10 \%$. L'expérimentateur doit se fixer une limite inférieure (ici $0,5 \mathrm{cc}$ correspondant à une erreur de $10 \%$ ) au-dessus de laquelle son dispositif expérimental ne lui permet pas de descendre s'il désire que l'erreur ne dépasse pas les limites acceptables. Pour la mesure d'un métabolisme plus faible, il doit utiliser un volume d'eau plus faible et éventuellement une méthode plus fine.

Parmi les méthodes que nous avons expérimentées, nous avons constaté que la méthode chimique, lorsque nous utilisions des pipettes de LEvY de $120 \mathrm{cc}$, permet de mesurer avec une bonne précision une consommation de $30 \mathrm{~mm}^{3} \mathrm{~d}^{\prime} \mathrm{O}_{2}$, soit le métabolisme horaire d'une grosse larve de l'Odonate Aeschna. Les seringues de vaN DAM permettent de mesurer un métabolisme de $5 \mathrm{~mm}^{3}$, soit le métabolisme horaire d'une Aselle (Crustacé Isopode), d'une grosse Planaire, ou d'une larve d'Ecdyonurus (Ephéméroptère). La méthode volumétrique de ScholANDER, telle que nous l'avons employée, permet de mesurer un métabolisme horaire de $10 \mathrm{~mm}^{3} \mathrm{~d}^{\prime} \mathrm{O}_{2}$.

Il convient donc de choisir la méthode la plus adaptée à la taille de l'animal ou des groupes d'animaux que l'on veut étudier.

Résumons les autres points qui entrenten ligne de comptedans le choix d'une méthode. Nous commencerons par les conditions offertes à l'animal.

Il faut que l'eau soit agitée ou stagnante suivant le biotope d'origine du sujet. Le respiromètre de Scholander a une hélice. On peut en introduire une dans le flacon de la méthode de WINKLER. Dans la méthode de SAYLE pour mesurer le $\mathrm{CO}_{2}$, les bulles d'air se dégageant dans le milieu agitent l'eau.

Il faut que la reconstitution grossière du substrat soit possible pour l'étude des animaux pétricoles ou fouisseurs. Seules les méthodes manométriques de WARBURG et BARCROFT l'interdisent.

Il faudrait maintenir autant que possible la constance du taux d'O $\mathrm{O}_{2}$ et de $\mathrm{CO}_{2}$, ce que permettent les méthodes en milieu renouvelé, la méthode de SchOLANDER et ce que permettrait une méthode de mesure du $\mathrm{CO}_{2}$ en circuit ouvert.

L'élimination des déchets autres que le $\mathrm{CO}_{2}$ serait également souhaitable. Elle n'est réalisée que dans les méthodes en milieu renouvelé (adaptation de la méthode chimique de WINKLER ou de la méthode polarographique).

Il faut enfin pouvoir régler la température et éventuellement le 
taux $\mathrm{d}^{\prime} \mathrm{O}_{2}$. L'appareillage doit donc pouvoir être contenu dans un réfrigérateur permettant d'opérer de 0 à $20^{\circ}$ et, pour les respiromètres contenant une phase gazeuse, il doit y avoir un moyen d'influencer la composition de cette phase (circulation de gaz dans l'appareil, comportant une entrée et une sortie).

Examinons ensuite le point de vue de l'expérimentateur.

Il peut être nécessaire d'avoir un appareil portatif, si l'on veut opérer directement dans le milieu naturel. La seringue de Fox \& WINGFIELD (1938) et sa modification par WHITNEY (1938) présentent un encombrement minimum et sont susceptibles d'être utilisées à l'extérieur.

Il peut être utile d'obtenir un enregistrement automatique pour suivre de façon continue les variations du métabolisme, de jour comme de nuit. Les seules méthodes permettant cet enregistrement sont le plongeur de Brown et une adaptation du polarographe (BRIGGS, Dyke \& KNowles 1959).

Enfin certaines techniques sont plus rapides que d'autres. Une fois mis en marche, les appareils manométriques ne demandent qu'une lecture des niveaux liquides et un calcul simple pour chaque résultat. Ces appareils sont donc avantageux lorsqu'il s'agit de suivre les variations du métabolisme dans le temps. Les respiromètres de Brown, WARBURG et BARCROFT peuvent être installés par batteries dans un même bac, donnant de nombreux résultats simultanés. Par contre, l'appareil de SCHOLANDER, avec son moteur et sa burette accessoire, est plus volumineux; on peut donc plus difficilement en installer un grand nombre. La méthode chimique est la plus longue, celle qui fournit le moins de résultats à un opérateur solitaire pendant un temps donné.

Aucune méthode ne remplit donc toutes les conditions qu'on peut demander. C'est à l'expérimentateur de choisir celle qui lui permet de jouer sur les facteurs qu'il tient pour les plus importants dans chaque cas: substrat, agitation de l'eau, taux d'O $\mathrm{O}_{2}$ et de $\mathrm{CO}_{2}$, température, élimination des déchets, appareil à enregistrement automatique, portatif, ou susceptible de fournir un grand nombre de mesures à la fois.

\section{RÉSUMÉ}

L'article comporte un rappel des principales méthodes, de leurs avantages et de leurs inconvénients. Les conditions faites à l'animal pendant l'expérience sont très variables d'une méthode à l'autre. Or des mesures comparatives effectuées par une méthode chimique et une méthode manométrique ont donné des résultats significative- 
ment différents. Les chiffres fournis par une seule méthode ne sont donc pas valables dans l'absolu. Lorsqu'on s'intéresse au métabolisme d'un animal dans la nature, il faut choisir une méthode où les conditions imposées déforment les résultats le moins possible.

\section{SUMMARY}

The principal methods for measuring the respiratory metabolism of aquatic animals are reviewed with their advantages and disadvantages. An animal does not undergo the same treatment in each of them. A chemical method and a manometric method were compared here and the results were found to differ, the former method yielding significantly lower values for Crenobia alpina and Dendrocoelum lactaeum and significantly higher values for Planaria gonocephala and Cloëon dipterum. The greatest caution is therefore necessary when basing a conclusion upon measures gathered by means of two different methods. Also when information on the normal metabolism of an animal is required, such a method must be selected as does not falsify the results by submitting the animal to grossly unnatural conditions.

\section{BIBLIOGRAPHIE}

AмBüHL, H. - 1959 - Die Bedeutung der Strömung als ökologischer Faktor. Schweiz. Z. f. Hydrol., XXI, 133-264.

Berg, K., Lumbye, J. \& OCKelmanN, K. W. - 1958 - Seasonal and experimental variations of the oxygen consumption of the Limpet Ancylus fluviatilis (O. F. Müller). F. exp. Biol., XXXV, 43-73.

BLÄsING, I. - 1953 - Experimentelle Untersuchung über den Umfang der oekologischen und physiologischen Toleranz von Planaria alpina Dana und Planaria gonocephala Dugès. Zool. Fb. (Abt. allg. Zool. Physiol.), LXIV, 112-152.

Briggs, R., Davies, F. S., Dyke, G. V. \& Knowles, S. G. - 1957 - Use of wide-bore dropping-mercury electrode and zinc reference electrode for continuous polarography. Chem. Ind., Feb. 23, 223-224.

BRIGGS, R., Dyke, G. V. \& KNOWLES, G. - 1958 - Use of the wide-bore dropping-mercury electrode for long-period recording of concentration of dissolved oxygen. Analyst, LXXXIII, 304-311.

BRIGGS, R., DYKe, G. V. \& KNOWLES, S. G. - 1959 - Electrical recorder for dissolved oxygen. Water and Waste Treatment F., Jan.-Febr.

BRown, F. A. JR. - 1954 - Simple, automatic, continuous-recording respirometer. Rev. Sci. Instr., XXV, 415-417.

BRown, F. A. JR - 1957 - Response of a living organism, under ,constant conditions" including pressure, to a barometric-pressure-correlated, cyclic, external variable. Biol. Bull., CXII, 288-304.

CALHOON, T. B. \& ANGERER, C. A. - 1953 - A volumeter for respiration of aquatic animals. Ohio f. Sci., LIII, 240-243.

Clausen, R. G. - 1936 - Oxygen consumption in fresh-water fishes. Ecol., XVII, 216-226. 
DAM, L. VAN - 1935 - A method for determining the amount of oxygen dissolved in $1 \mathrm{cc}$ of water. . exp. Biol., XII, 80-85.

Dixon, M. - 1951 - Manometric methods. Cambridge Univ. Press.

Dussart, B. \& Francis-Boeuf, C. - 1949 - Technique du dosage de l'oxygène dissous dans l'eau basée sur la méthode de Winkler. Circulaires $d u$ Centre de Recherches et d'Etudes Océanogr., I. T. $\mathrm{n}^{\circ} 1$.

EDWARDS, R. W. - 1958 - The relation of oxygen consumption to body size and to temperature in the larvae of Chironomus riparius Meigen. F. exp. Biol., XXXV, 383-395.

EwER, R. F. - 1942 - On the function of haemoglobin in Chironomus. F. exp. Biol., XVIII, 197-205.

Fox, H. M. \& Simmonds, B. G. - 1933 - Metabolic rates of aquatic Arthropods from different habitats. F. exp. Biol., X, 67-74.

Fox, H. M. \& WINGFIELD, C. A. - 1938 - A portable apparatus for the determination of oxygen dissolved in a small volume of water. $\mathcal{f}$. exp. Biol., $\mathrm{XV}, 437-445$.

Giguere, P. A. \& LAUzier, L. - 1945 - Analyse polarométrique de l'oxygène dissous avec u. e microélectrode de platine. Canad. F. Res., B, XXIII, $223-233$.

KroGH, A. - 1935 - Precise determination of oxygen in water by syringepipets. Ind. eng. Chem., VII, 131-133.

LALlEMAND, S. - 1932 - Sur l'efficacité d'une couche d'huile de paraffine utilisée comme agent de protection contre l'oxygène de l'air. $C . R$. Soc. Biol., CX, 719-721.

Lane, C. E., Tierney, J. Q. \& Hennacy, R. E. - 1954 - The respiration of normal larvae of Teredo bartschi Clapp. Biol. Bull., CVI, 323-327.

LONGMUIR, I. S. - 1954 - Respiration rate of Bacteria as a function of oxygen concentration. Biochem. F., LVII, 81-87.

LONGMUIR, I. S. - 1957 - Respiration rate of rat-liver cells at low oxygen concentrations. Biochem. F., LXV, 378-382.

LUND, E. J. - 1919 - A simple method for measuring carbon-dioxide produced by small organisms. Biol. Bull., XXXVI, 105-114.

MANN, K. H. - 1958 - Seasonal variation in the respiratory acclimatization of the leech Erpobdella testacea (Sav.). F. exp. Biol., 314-323.

Moore, E. W., Morris, J. C. \& OKUN, D. A. - 1948 - The polarographic determination of dissolved oxygen in water and sewage. Sewage Works, XX, 1041-1053.

Philipson, G. N. - 1954 - The effect of water flow and oxygen concentration on six species of Caddis fly (Trichoptera) larvae. Proc. zool. Soc. Lond., CXXIV, 547-564.

Pomeroy, R. \& Kirschman, H. D. - 1945 - Determination of dissolved oxygen. Proposed modification of the Winkler method. Industr. eng. Chem., XVII, 715-716.

Prat, H. - 1954 - Analyse microcalorimétrique des variations de la thermogénèse chez divers Insectes. Canad. $\mathcal{F}$. Zool., XXXII, 172-197.

Punt, A. - 1950 - The respiration of Insects. Physiol. comp. Oecol., II, $59-74$.

Punt, A. - 1956 - Further investigations on the respiration of Insects. Physiol. comp. Oecol., IV, 121-131.

RotTHaUWE, H. W. - 1958 - Die Sauerstoffbestimmung in See- und Süsswasser mit Hilfe der Quecksilbertropfelektrode und ihre Anwendung bei physiologischen Untersuchungen. Kiel Meeresforschung., XIV, 48-63. 
SAYLE, M. H. - 1928 - Factors influencing the rate of metabolism of Aeschna umbrosa nymphs. Biol. Bull., LIV, 212-230.

Scholander, P. F., Dam, L. van, ClafF, C. L. \& Kanwisher, J. W. 1955 - Micro-gasometric determination of dissolved oxygen and nitrogen. Biol. Bull., CIX, 328-334.

SCHOLANDER, P. F. \& EDWARDS, G. A. - 1942 - Volumetric microrespirometer for aquatic organisms. Rev. sci. Instr., XIII, 292-295.

SPOoR, W. A. - 1948 - Use of the dropping-mercury electrode for the continuous measurement of dissolved oxygen in flowing water. Science, CVIII, 421-422.

Theriault, E. J. - 1925 - The determination of dissolved oxygen by the Winkler method. U. S. Public Health Bull., CLI, 1-43.

Tobias, J. M. - 1943 - Microrespiration techniques. Physiol. Rev., XXIII, $51-76$.

WaLSHE, B. M. - 1947 - On the function of haemoglobin in Chironomus after oxygen lack. F. exp. Biol., XXIV, 329-342.

WAutier, J. \& PatteE, E. - 1955 - Expérience physiologique et expérience écologique. L'influence du substrat sur la consommation d'oxygène chez les larves d'Ephéméroptères. Bull. Soc. Linn. Lyon, $\mathrm{n}^{\circ} 7,178-$ 183.

WhITNEY, R. J. - 1938 - A syringe-pipette method for the determination of oxygen in the field. $\mathcal{F}$. exp. Biol., XV, 564-570.

WINKLER, L. W. - 1888 - Die Bestimmung des im Wasser gelösten Sauerstoffes. Ber. dtsch. chem. Ges., XXI, 2843-2854.

WINKLER, L. W. - 1924 - Bestimmung des gelösten Sauerstoffs mit kleinen Wassermengen. Z. Untersuch. Nahr. Genussm., XLVII, 257-259,

WooD, K. G. - 1953 - Polarograms of oxygen in lake water. Science, CXVII. $560-561$.

Laboratoire de Zoologie Générale

Faculté des Sciences de Lyon 